\title{
Development of Indian Coin based automatic shoe Polishing Machine using Raspberry pi with Open CV
}

\author{
D.Srihari ${ }^{1}$, B.Ravi Kumar ${ }^{2}$, K.Yuvaraj ${ }^{3}$ \\ Assistant Professor, Department of ECE, S V College of Engineering, Tirupati, A.P, India ${ }^{1,2,3}$
}

\begin{abstract}
Aimed at challenging the drawbacks of conventional shoe polishing machine operations, a new automatic shoe polishing machine system is designed for performing polishing

work instead of manual legs oscillation that is characteristic of low safety, low efficiency, long time and body stress problems. In this system proposed to design an automatic shoe polishing machine with portable coin box using Raspberry pi with open source computer vision library (openCV) as this image processing library tool has more advantages compared to the previous one used in the machine. The automated system replaced the single or specific coin system by denomination Indian coins like one rupee, two rupees, five rupees, ten rupees. When a put the coin into the coin box after that coin recognized by open CV with Scale Invariant Feature Transform (SIFT) keypoint detector and Fast Library for Approximate Nearest Neighbor (FLANN) keypoints matcher algorithm which detects the coin and instructs to the raspberry pi board also control the polishing facilities. This system can be located at stations for Airports, Hospitals, and Bus stands. Further, located at institutions and industrials.
\end{abstract}

KEYWORDS: shoe polishing, open CV, Raspberry pi, SIFT, portable coin box.

\section{INTRODUCTION}

"Cleanliness is next to godliness" is a famous proverb which says cleanliness is the way to godliness and without cleanliness we cannot get godliness. Today, most people work in industries, hospitals, airport, assembly on the chemical side, IT companies etc., so they should wear shoes. Once the person comes out of his home into the outside environment which is highly polluted, dust particles get deposited on the shoe. The person finds it difficult to clean the shoe every time it gets dirty. Also, in manual cleaning of the shoes, if the wire of the brushes is too hard and if the cleaner handles it without care, it may damage the leather of the shoe and decreases its life. Now a days shoe polishing machine operated by individual plugs into the machine used for home and coin based machine used for public place. In coin based shoe polishing machine has only used as specific or fixed coin. To overcome this, the proposed method named "Automatic shoe polishing machine using Raspberry pi with OpenCV" is aimed at designing a portable machine with coin box which cleans the shoe in less time without any human assistance. In this machine operated by all Indian coin models like `1, `2, `5, `10 and remain balance will be provided by the machine. The survey of various papers that have contributed in the SIFT feature extraction, FLANN [12] algorithm for matching of live coin image and reference coin image using open CV [11] image processing library tool. David Lowe proposed a new approach to extracting distinctive invariant features from images to perform real time object recognition using SIFT [1], [2] and [3] algorithm. When a capturing the coin image does not clear due to lighting variation. In this problem avoided by using SIFT flow [4] and [5] method. The feature extraction [9] is a very important of part in computer vision for extracting features in coin image. The reference coin image and live coin image particular feature point should be correlated by using normalized cross-correlation coefficient (NCC) [6] and [7] with SIFT algorithm. The further of this paper is structured as follows. Section II illustrate the existing of automatic shoe polishing machine and how will be it overcome by the drawbacks. Section III describes the proposed work. In this section fully coin reorganization and detection using SIFT algorithm with openCV also every hardware components have explained. Section IV is a results and discussions. In this section, how will be recognized coin and shows the hardware circuit in proteus simulation results. Finally a conclusion and future enhancements are given in the section V.

\section{EXISTING WORK}

In existing method, shoe polishing machine by using only single or specific coin based on sensors equipment method. The job of the sensors is to measure physical properties of the coins, such as dimensions, conductivity, magnetic permeability, elasticity, etc. there are many sensors used in polishing machine like optical sensors, impact sensors, electromagnetic sensors, acoustic sensors which sensors can be used to coin recognition for automatic shoe polishing 
machine. The main drawbacks of the existing system has access the significant or particular coins. So we need to that particular coin on the spot. To overcome this, our proposed system can access by all Indian coin models.

\section{PROPOSED WORK}

"Fig. 1", shows as proposed algorithm. The proposed work is aimed to design an Automatic shoe polishing machine using embedded systems. In this Raspberry pi based shoe polishing machine supports multiple of different denomination all Indian coins like one rupee, two rupees, five rupees and ten rupees. Keep the coin valid numerical character front of you after that put into the coin box. Then the machine has recognized the coins and then coins will be accounted. Accounts have sufficient to shoe polishing, and may not be enough to account again coins put into the coin box and verified accounted. If the shoe polishing pay equals to accounts then motor will be turn

on. After some time period motor will be turned off. The accounts have higher than polishing pay and remain balance will be provided by the machine. In this paper, we are going to create a compact machine using embedded Linux development board and using open computer vision environment.

\section{A. CAMERA MODULE}

The Raspberry pi camera module is capable of taking full

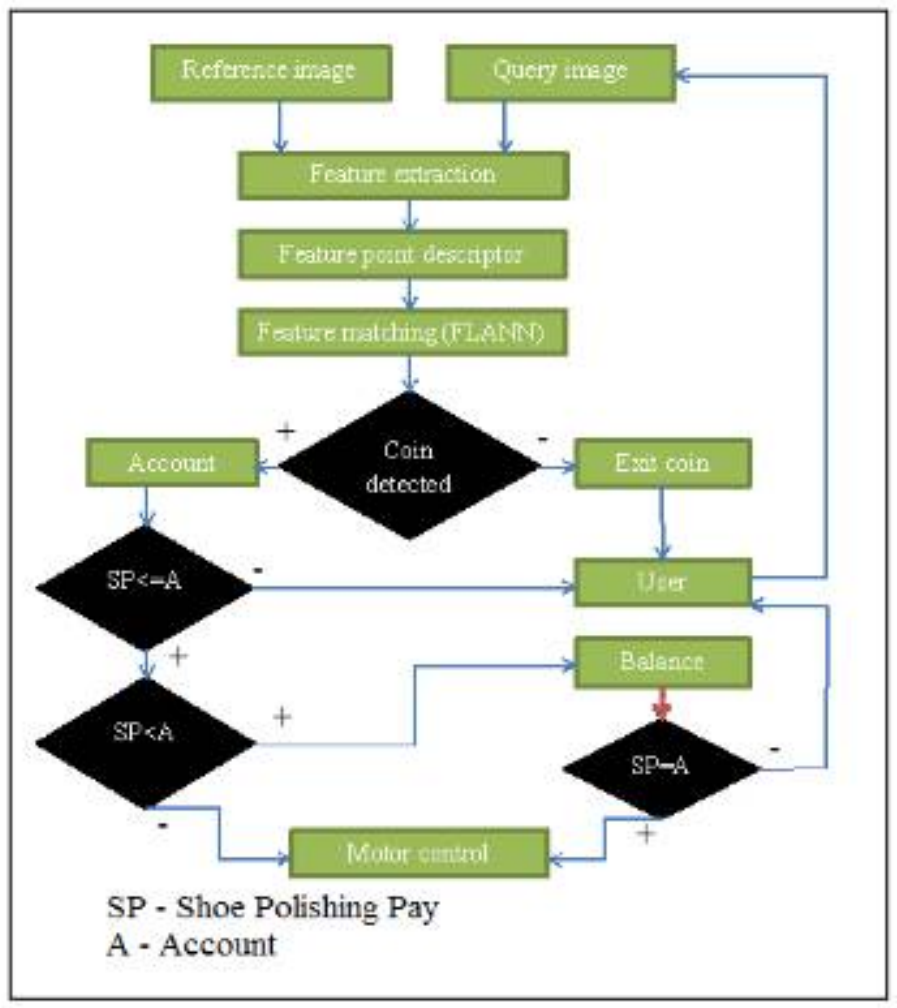

Figure 1. Proposed algorithm

HD 1080p photo and video can be controlled programmatically. In this module only capturing the arrival of coin image that coin image stored will be in the corresponding file location.

\section{B. COIN SELECTOR}

It's a preprocessing of coin recognition and detection using openCV with SIFT keypoint detector and FLANN matcher [12] in Raspberry pi processor. Coin recognition in computer vision is a task of finding given coin in an image or video sequence. Coin sensors recognize a multitude of coins in images with little try, despite the fact that the image of the coins may be varied somewhat in different viewpoints, in much different size of coin scale or even when they are translated or rotated. A coin can even be recognized when they are partially obstructed from view. This work is still an opposition for computer vision systems in general. David Lowe [1], [2] and [3] pioneered the computer vision approach to extracting and using scale-invariant SIFT features from images to perform reliable object recognition. SIFT keypointsof objects are feature extracted from a set of coin reference images and stored in the database. Simultaneously SIFT keypoints of objects are feature extracted [9] from a new arrival of coin image. "Fig. 2", shows how offset issues are resolved.In this paper. 


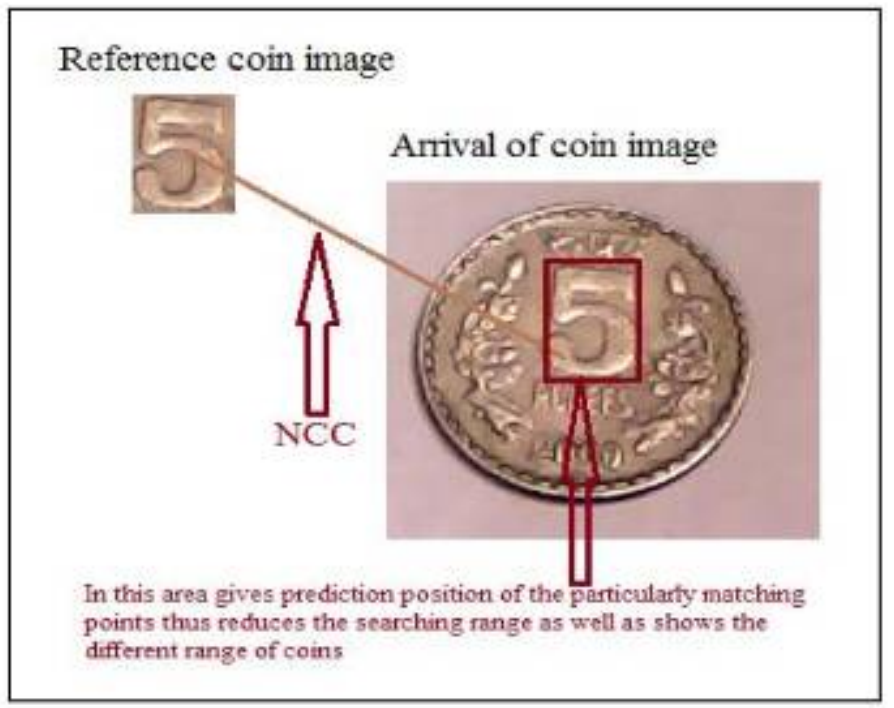

Figure 2. The Correlation between the reference coin image and arrival of coin image using NCC

which can be used to normalized cross-correlation coefficient method [6] and [7]. NCC method has to separate the reference coin image and arrival of coin image. Then calculate the crosscorrelation coefficient according to the both coin images. In this job of quickly coin recognition in the raspberry pi processor. The reference coins image recognized in a new arrival of coin image by individually comparing each feature from the new coin image to this database and finding candidate matching features based on Euclidean distance [6] of their feature vectors. Next problems are lighting variations that particularly affect the coin surface appearance. In this problem is does not make coins comparison. An example of this lighting variation is given the "Fig. 3", where both images of the same coin samples, but different lighting variation is shown. To overcome this, the proposed method called as SIFT flow method [4] and [5].

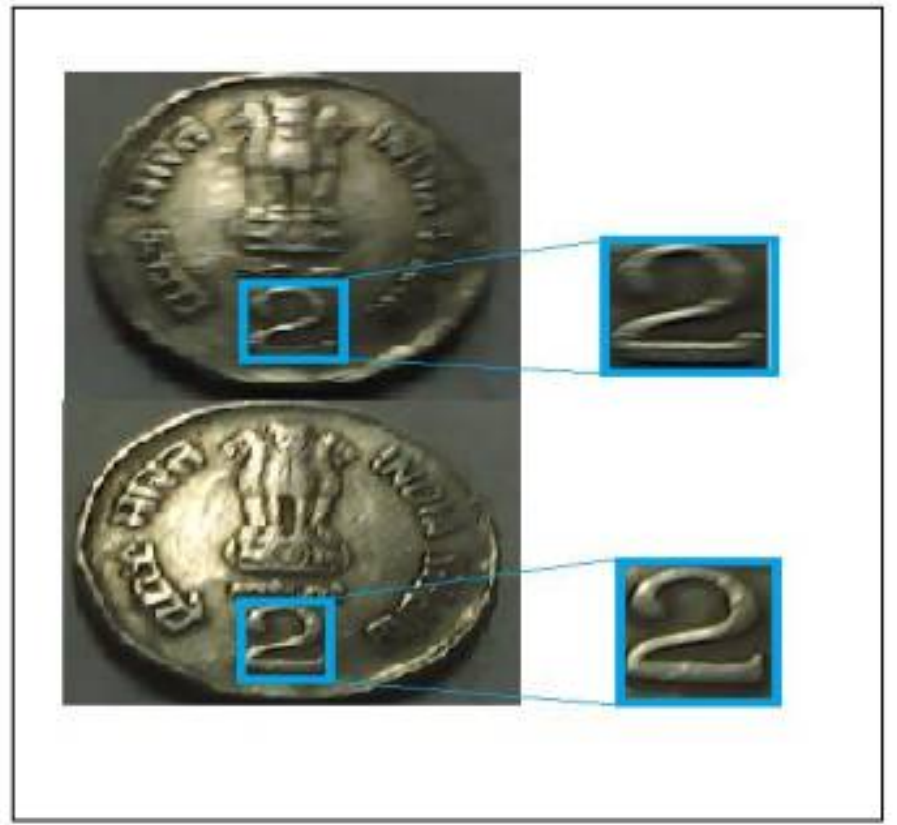

Figure 3. Two images of the same coin brighten from different Directions

Figure 1. Raspberry pi board Raspberry pi depicted shown in "Fig 4", It's a capable of 


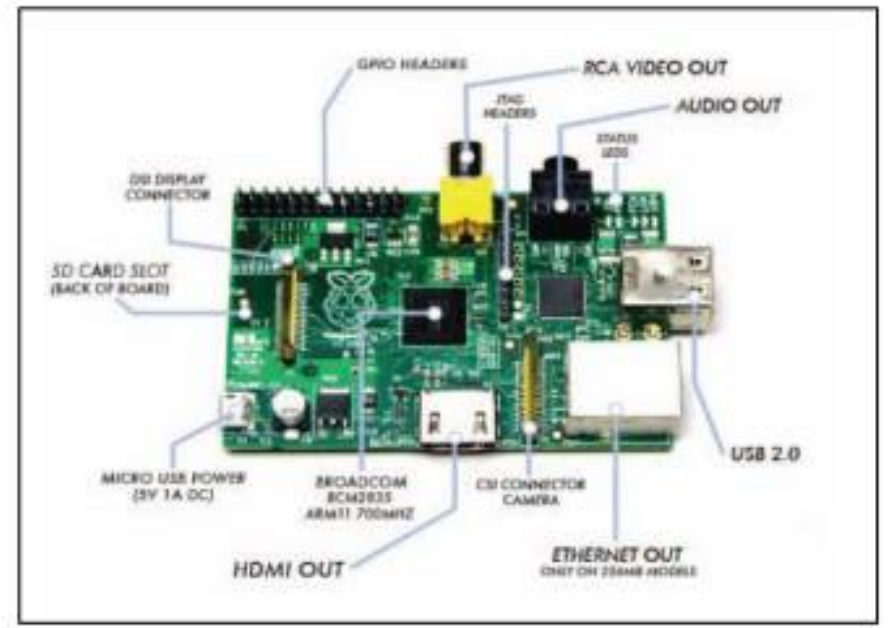

Figure 4. Raspberry pi board

small computer which can be used in real time application projects or individually electronics products and for much more of the thinks that your desktop or laptop does, like showing films, editing Photoshop, listening music, high definition video game, internet browsing etc... The Raspberry pi [13] processor is a low cost, palm size computer that plugs into a computer or Television monitor, and uses a standard mouse and keyboard. Raspberry pi based on Broadcom

BCM2836 Arm7 Quad Core Processor single board computer running at $900 \mathrm{MHz}$ Its consist of 1GB Ram,40 pin extended GPIO,4 USB ports,4 pole stereo output and composite video port. it has made an easy connection between monitor and HDMI port. Micro SD port for loading your operating system (Raspbian OS) and storing data and micro USB power source available.

\section{POWER SUPPLY}

Generally, Every electrical and electronic product that we use in our day to day life so need to require a power supply. In general, we can use an AC supply of $230 \mathrm{~V} 50 \mathrm{~Hz}$, but this power has to be converted into the required form with required values for providing power supply. In our system has only $5 \mathrm{~V}$ DC for the raspberry pi board. So we go for a step-down converter. It's used for converting the high voltage into low voltage. $230 \mathrm{~V}$ AC is converted into $12 \mathrm{~V}$ AC using a stepdown transformer. Then $12 \mathrm{~V}$ AC rectified into $5 \mathrm{~V}$ DC using electronic components like rectifier, filter and regulator.

\section{E. INFRARED SENSORS}

Automatic shoe polishing machines operate with infrared sensors and can detect the presence of a shoe in the slot. When the sensor is activated, a soft brush of high quality fiber starts rotating and cleans the shoe within few seconds.

F. Single Phase Induction Motor with Soft Brush

Single phase induction motor (SPIM) is an AC motor an electrical energy converted into mechanical energy to perform any physical task (rotated with brush). In this motor accommodate to shoe polishing machine with low cost, better reliability, eases to repair, and better maintenance when compare to the other motors like high expensive motor. This motor controlled by PWM AC chopper control [10] for energy saved.

\section{RESULTS AND DISCUSSIONS}

In this section, present an automatic shoe polishing machine using Raspberry pi with openCV is an integrated automatic system consists of software and hardware. The first main part is coin recognition and detection using SIFT algorithm with the openCV [11] image processing tool. In

order to test the validity of all Indian coin images, this paper applied a real time sample of images. The arrival of coin image pixel size is $500 \times 450$ and reference coin image size is depends upon the cropped numerical character size like 1, 2,5 , and 10. It's more over 50 samples taken [6].

\section{A. FEATURE EXTRACTION OF COIN IMAGES}

"Fig. 5", shows the feature extracted from reference coin image and live coin image. The images taken by the pi cameras have high resolution. Its size is approximately 5 megapixels. In this way is a processing of system time period takes large. In order to reduce the system processing time period, we will give up image preprocessing and coin recognition process. We first of all coin images taken by the pi camera after that the first coin frame image taken in a 
corresponding file location. The first coin frame image read from file, after that coin frame image converted into gray scale for reduced memory size. Then gray scale image has been extracted features using the SIFT algorithm.

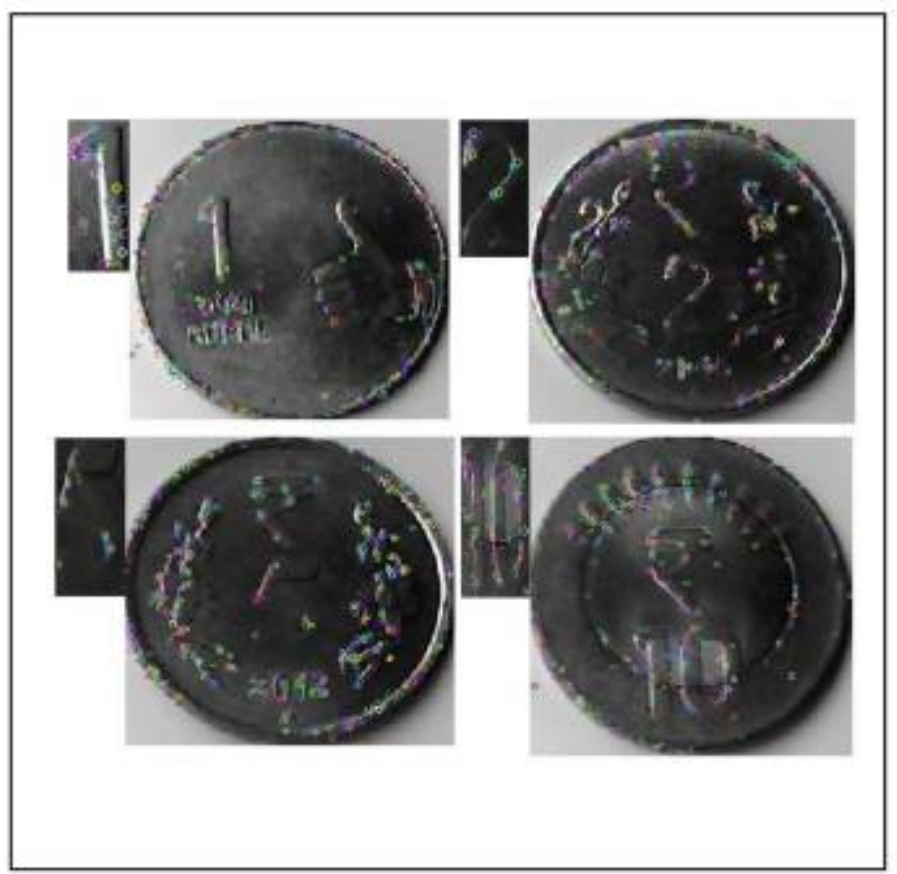

Figure 5. Key points or features extracted from coin image using SIFT algorithm

\section{B. RECOGNIZED COIN IMAGE}

"Fig. 6", shows the one rupee and two rupees coin recognition. On the left side query coin image has 998 keypoints detected and the reference coin image has 27 keypoints detected. Both coin images has 27 keypoints or features matched, so the corresponding coin will be detected.Then right side query coin image has 225 keypoints detected and the reference coin image has 31 keypoints detected. Both coin images has 30 keypoints or features matched, so the corresponding coin will be detected.

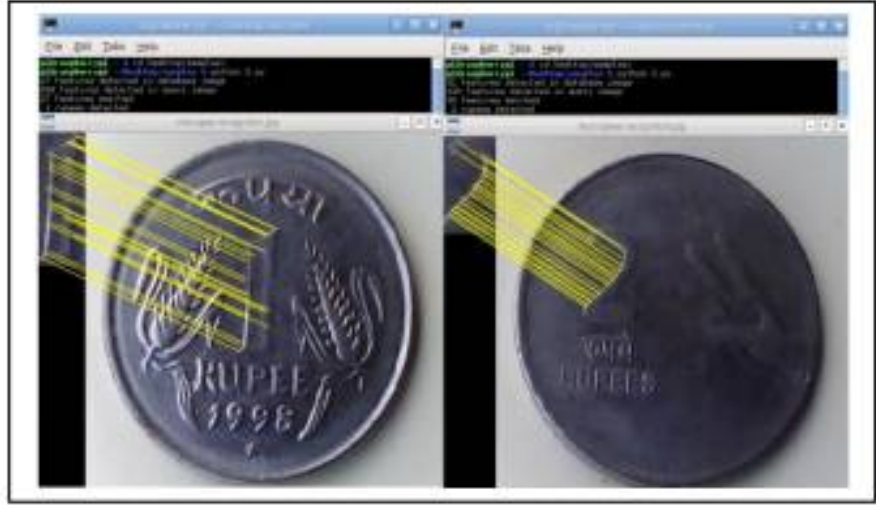

Figure 6. The example of lines has to SIFT feature points matched between reference coin image and live coin image (left-1 rupee coin recognition and right 2 rupees coin recognition)

"Fig. 7", illustrates the image on SIFT feature points matched between reference coin image and live coin image and test image with an angular difference of rotated left 90 and rotated right 900

between them. Where the left corner side image has ten rupees coin recognition with rotating right. The query coin image has 333 keypoints detected and the reference coin image has 41 keypoints detected. Both coin images has 37 keypoints or features matched, so the corresponding coin will be detected. The right corner side image has ten rupees coin recognition with rotating left. The query coin image has 333 keypoints detected and the reference coin image has 41 keypoints detected. Both coin images has 37 keypoints or features matched, so the corresponding coin will be detected. 


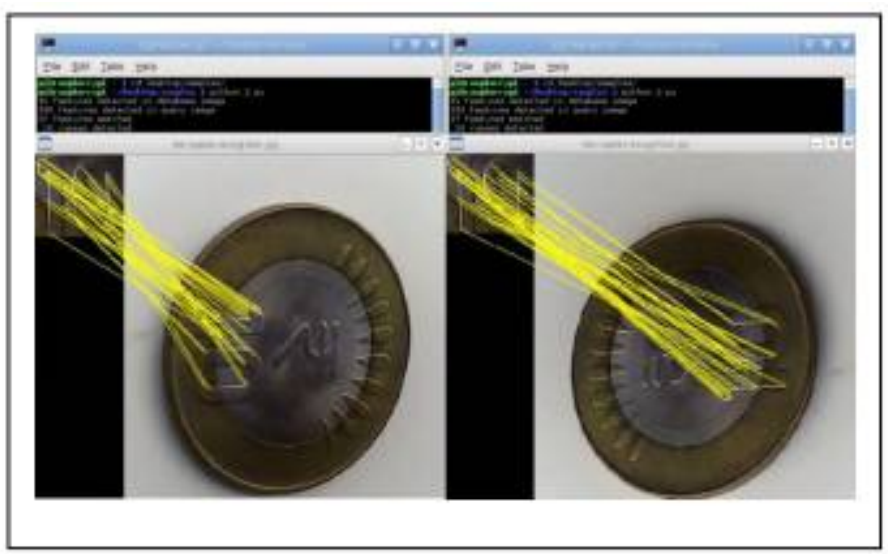

Figure 7. The example of lines has to SIFT feature points matched between reference coin image and different angle of live coin image (10 rupees coin recognition)

Table I shows a comparison between the live coin features (LCF) and reference coin features (RCF) after that corresponding feature matched using modified SIFT algorithm and also different kinds of Indian coin models compared. In our proposed SIFT algorithm has improved coin selection process and also reduced by the execution time period when compared to the normal SIFT algorithm [1], [2] and [3].

TABLE I. Comparison of LCF and RCF Matching Time

\begin{tabular}{|c|c|c|c|c|c|}
\hline Coin models & LCF & RCF & $\begin{array}{c}\text { Matched } \\
\text { features }\end{array}$ & $\begin{array}{c}\text { Normal } \\
\text { SIFT } \\
\text { matching } \\
\text { Time(Sec) }\end{array}$ & $\begin{array}{c}\text { Improved } \\
\text { SIFT } \\
\text { matching } \\
\text { Time(Sec) }\end{array}$ \\
\hline 1 (model 1) & 998 & 27 & 27 & 21 & 14 \\
\hline 1 (model 2) & 510 & 40 & 35 & 19 & 11 \\
\hline 2 (model 1) & 225 & 31 & 30 & 13 & 8 \\
\hline 2 (model 2) & 250 & 21 & 19 & 13 & 9 \\
\hline 2 (model 3) & 404 & 13 & 10 & 16 & 10 \\
\hline 5 (model 1) & 519 & 12 & 12 & 17 & 12 \\
\hline 5 (model 2) & 402 & 13 & 10 & 17 & 11 \\
\hline 5 (model 3) & 384 & 34 & 32 & 14 & 8 \\
\hline $\begin{array}{c}10 \text { rotated } \\
\text { left }\end{array}$ & 333 & 41 & 37 & 14 & 7 \\
\hline $\begin{array}{c}\text { 10 rotated } \\
\text { right }\end{array}$ & 333 & 41 & 37 & 15 & 9 \\
\hline
\end{tabular}

\section{SIMULATION CIRCUITS OF SHOE POLISHING SYSTEM}

The proposed shoe polishing simulation circuit show in "Fig.8", in this circuit the connection is made between raspberry pi board and pic microcontroller. The Raspberry pi processor recognition the coin and detected through the corresponding Raspberry pi GPIO pins and pic microcontroller get input according to the GPIO pins. The GPIO 7th pin detects only one rupee coin and it is connected to the input of RB0 (33). Similarly, two rupee coin detected (GPIO11, RB0-34) five rupee coin detected (GPIO-13, RB035) ten rupee coin detected (GPIO-15, RB0-36) in this simulation circuit consider the corresponding GPIO output instead of the corresponding switch. It contains four switches each switch has different denomination of coin identification.

Say for example shoe polishing charge is 5 rupees. Shoe polishing machine ready when account is 5 or above 5 . The account has above 5 rupees and remain balanced will be provided. 


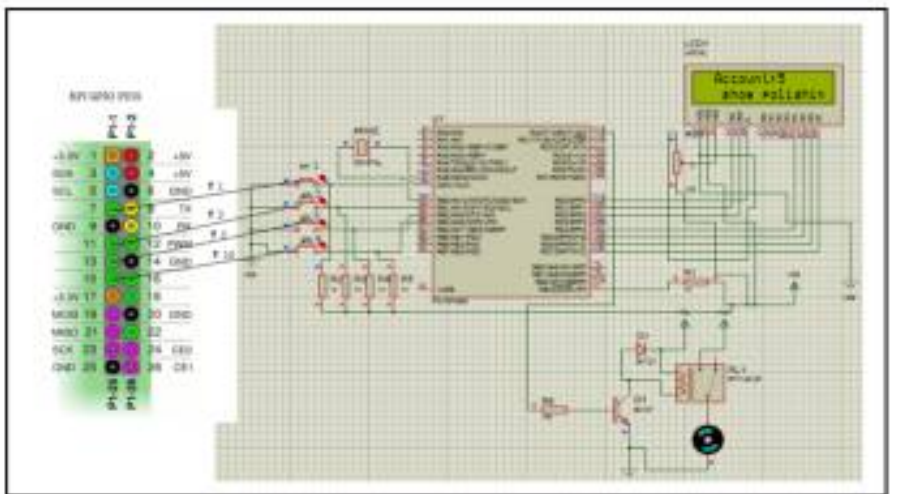

Figure 8. The example of Simulation circuit in shoe polishing System

\section{CONCLUSION}

In this paper a novel method of coin based automatic shoe polishing machine using raspberry pi processor and openCV image processing tool [11] has been designed and developed. The main part of the coin recognition process is employed by installing openCV library into the raspberry pi processor [8]. SIFT algorithm has detected the keyponits and extract keypoints finally, FLANN [12] algorithm provides matching keypoints. Hence this method provides all Indian coin

recognized rather than the expensive coin sensor module. An automatic shoe polishing machine with portable coin box is a complete process produced for polishing the shoe in order to control the environment and also it gives the style of the persons. The openCV image processing tool is used for avoiding fake coin or invalid coin. Thus we can conclude from the above that the usage of shoe polishing machine is a need for all the industrial employees and institutions like school and college zones. In future enhancement, RFID or Debit card based automatic shoe polishing machine designed and implemented in product.

\section{REFERENCES}

[1] D.G. Lowe, “Object recognition from local scale invariant features,” IEEE Conf. vol. 2, pp. 1150-1157, 1999.

[2] D.G. Lowe, "Local feature view clustering for 3D object recognition," IEEE Conf. vol. 1, pp. 682-688, 2001.

[3] D.G. Lowe, "Distinctive Image Features from Scale-Invariant Keypoints," International Journal of Computer Vision. vol. 60, pp. 91110, January 2004.

[4] C. Liu, J. Yuen, and A. Torralba, "SIFT Flow: Dense Correspondence across Scenes and Its Applications,” IEEE Transl. vol. 33, issue. 5, pp. 978-994, 2011

[5] C. Liu, J. Yuen, and A. Torralba, "SIFT Flow: Dense Correspondence across Scenes and Its Applications," In European Conference on Computer Vision (ECCV), 2008

[6] F. Zhao, Q. Huang,W. Gao, “Image Matching By Normalized Cross Correlation,” IEEE Conf. Vol. 2, pp. 729-732, 2006.

[7] W. Wantong, L. Yuling, Z. Qingliang, "A New Approach to Automatic Feature Based Registration of Multi Sensor Remote Sensing Images," IEEE Conf. pp. 1-5, 2012.

[8] S. Sivaranjani, S. Sumathi, "Implementation of Fingerprint and Newborn Footprint Feature Extraction on Raspberry Pi," IEEE Conf. pp. 1-6, 2015.

[9] F. Cao, R. Wang, L. Zhang, "Feature extraction and stereo matching algorithm for lunar surface,” IEEE Conf. pp. 1399-1402, 2011.

[10] V. Thanyaphirak, V. Kinnares, A. Kunakorn, "PWM AC chopper control schemes for energy saving of single-phase induction motors," IEEE Conf. pp. 82-87, 2012

[11] I.Culjak, D. Abram, T. Pribanic, H. Dzapo, M. Cifrek, “A brief introduction to OpenCV,” IEEE Conf.pp. 1725-1730, 2012.

[12] W. Budiharto, "Robust Vision-based detection and grasping object for manipulator using SIFT keypoint detector," IEEE Conf. pp. 448-452, 2014.

[13] A. Bradbury, B. Everard, "Learning Python with Raspberry Pi,” WILEY, 2014. 